\title{
LUDOS: uma Infraestrutura para Gamificação em Ecossistemas de E-learning
}

\author{
Welington Veiga, Fernanda Campos, Regina Braga, José Maria David
}

Universidade Federal de Juiz de Fora - Programa de Pós-graduação em Ciência da Computação - Núcleo de Pesquisa em Engenharia do Conhecimento

Juiz de Fora - MG - Brasil

welington.veiga@ice.ufjf.br, fernanda.campos@ufjf.edu.br,

Regina.braga@ice.ufjf.br, jose.david@ufjf.edu.br

\begin{abstract}
This paper presents LUDOS, an infrastructure based on web services to add gamification to E-learning Ecosystem. Data collected from the interaction of students with Learning Objects (LO) and the rewards obtained, such as points and medals, allow the creation of social profile pages with achievements, leaderboards and individual and collective goals monitoring. The goal is to promote students' engagement and motivation.
\end{abstract}

Resumo: O presente trabalho apresenta a LUDOS, uma infraestrutura baseada em serviços web para adicionar gamificação a Ecossistemas de Elearning. São coletados dados a partir da interação dos estudantes com Objetos de Aprendizagem (OA) e as recompensas obtidas, como pontos e medalhas, permitem a criação de páginas de perfis sociais com histórico de conquistas, quadros de líderes e acompanhamento de metas individuais e coletivas. O objetivo é favorecer o engajamento e a motivação dos estudantes.

\section{Introdução}

O uso de ambientes e-learning é uma realidade, e seu crescimento é evidenciado pelo número de cursos a distância oferecidos no ensino superior [Sridharan et al 2010] [Docebo 2014]. Hoje esses ambientes de e-learning não são apenas repositórios de conteúdo, mas ambientes virtuais completos para ensino e aprendizagem [Del Blanco et al 2011]. O uso de tecnologias para integrar diferentes ferramentas educacionais, a grande diversidade de atores e novos modelos de interação têm reforçado a perspectiva de Ecossistemas de E-learning, que considera em uma comunidade de ensinoaprendizagem à distância: conteúdo, princípios e métodos, sistemas e processos, além de recursos de gerenciamento de aprendizagem [Sridharan et al 2010]. Esses ecossistemas possuem ainda níveis de interação entre comunidades, grupos e indivíduos [Chang e Guetl 2007]. Sob essa perspectiva, o reuso e compartilhamento de recursos educacionais, como Objetos de Aprendizagem (OA), continua sendo um fator fundamental.

Do ponto de vista pedagógico, a motivação continua sendo uma das limitações do e-learning, uma vez que é difícil transmitir emoção ou engajar os estudantes da mesma forma que em um ambiente presencial [Muntean 2011] [Glover 2013]. A gamificação, ou seja, o uso de características de jogos fora do contexto de jogos, adequa-se particularmente bem a ambientes de e-learning e OAs e tem despertado um crescente interesse de pesquisa, motivado inclusive pelos resultados de sua aplicação em outros domínios [Borges et al 2014]. 
A grande variedade de plataformas é uma das características do domínio de elearning [Docebo 2014], tornando o esforço para a adaptação dessas plataformas, para inclusão de características de jogos em larga escala em Ecossistemas de E-learning, um desafio. Neste trabalho é apresentado a LUDOS, uma infraestrutura baseada em serviços web, que facilita o uso de gamificação em Ecossistemas de E-learning, estendendo os OAs distribuídos no ecossistema para que possam enviar dados de conquistas obtidas pelos alunos e permitindo que esses dados sejam utilizados para oferecer recursos que favoreçam o engajamento e a motivação no ecossistema.

Esse trabalho pretende ainda contribuir com o projeto BROAD [Nery et al 2012] [Campos et al 2012][Pereira et al 2012] que engloba pesquisas relacionadas a investigação e adoção de tecnologias de software, tais como ontologias, serviços web semânticos, agentes, sistemas de recomendação e redes sociais para construir uma arquitetura para a busca personalizada por OAs, bem como para a sua composição em conteúdos educacionais. Dessa forma, este trabalho pretende evoluir a proposta do projeto $\mathrm{BROAD}$, ampliando a visão do processo de recomendação de objetos de aprendizagem para a inclusão de características de gamificação, em um modelo de Ecossistemas de E-learning.

Este artigo está assim organizado, além desta introdução: a seção 2 discute Ecossistemas de E-learning e gamificação, e na seção 3 são apresentados os trabalhos relacionados. Na seção 4 é descrita a infraestrutura LUDOS, sua arquitetura e as possibilidades de integração com Ecossistemas de E-learning. Na seção 5 é apresentado o protótipo desenvolvido, o processo de desenvolvimento e uma avaliação do modelo de gamificação. Na seção 6 estão as considerações finais e trabalhos futuros.

\section{Referencial Teórico}

Um Ecossistema de E-learning é uma perspectiva de análise de um ambiente de ensinoaprendizagem por meio eletrônico que compreende diferentes fatores, em uma abstração dos ecossistemas biológicos. Guetl e Chang (2008) classificam ecossistemas digitais do domínio educacional em componentes bióticos (professores, instrutores, tutores, estudantes, pedagogos, especialistas) e abióticos (multimídia, software, tecnologia, equipamentos tradicionais), assim como as interações entre eles em um ambiente dentro de determinada fronteira, estabelecidas por pressões internas e externas (evolução do conhecimento, objetivos educacionais e aspectos culturais).

Muitas vezes um Ecossistema de E-learning possui uma plataforma a partir da qual outras ferramentas, serviços e recursos que podem ser de diferentes fornecedores se integram. Essa plataforma pode ser composta por ferramentas como Sistemas de Gestão de Conteúdos de Aprendizagem (LCMS), Sistemas de Gestão da Aprendizagem (LMS) e Sistemas de Distribuição de Conteúdo (CDS) [Guetl e Chang 2008], e sob essa perspectiva é de interesse de pesquisa não somente os aspectos técnicos relacionados a essas ferramentas, mas também as possibilidades de integração, distribuição de conteúdo educacional, as diferentes comunidades de atores e suas interações entre atores e com a plataforma.

O termo gamificação originou-se na indústria de mídias digitais, tornando-se amplamente utilizado a partir de 2010, e se refere ao uso de elementos de jogos como estética, mecânica e dinâmica em contextos não relacionados a jogos, para influenciar, engajar e motivar pessoas [Kapp 2012][Medeiros et al 2014]. No domínio educacional, o uso de jogos não é novo, mas recentemente têm despertado crescente interesse [Kapp 2012][Domínguez et al 2013][Borges et al 2014]. 
Camadas de software de "gamificação" frequentemente oferecem recursos como recompensas e sistemas de reputação como pontos, emblemas, medalhas, níveis, quadro de líderes, prêmios virtuais e avatares [Amriani et al 2013][Dicheva et al 2015]. É importante ressaltar que, além das soluções tecnológicas necessárias, as idéias e conceitos de gamificação devem estar presentes durante o projeto de conteúdo e iniciativas educacionais [Domínguez et al 2013] a fim de promover o engajamento e a motivação dos alunos em sintonia com os objetivos educacionais definidos.

\section{Trabalhos Relacionados}

Estão relacionados a esta pesquisa trabalhos que tratam da integração de ferramentas e serviços externos a ambientes de e-learning, onde a integração e interoperabilidade de serviços fornecidos por ferramentas externas e as plataformas é complexo e ainda não possui uma solução genérica [Alario-Hoyos et al 2013]. Existem iniciativas de padronização da integração como o IMS Learning Tools Interoperability (LTI) [IMS GLC 2006] que possui duas versões: Full LTI, que define um contrato complexo para uma integração total entre uma ampla variedade de ferramentas, e o Basic LTI que possui um mecanismo de integração simplificado, embora limitado. O GLUE! [AlarioHoyos et al 2013] é uma arquitetura que busca diminuir o esforço para o desenvolvimento necessário na integração de ferramentas desenvolvidas em diferentes tecnologias com diferentes plataformas através de uma arquitetura de provê adaptadores específicos para cada ferramenta e plataforma.

Para integração de diferentes componentes e ferramentas em Ecossistemas de Elearning, serviços web são amplamente utilizados [Doelitzscher et al 2011] [Lohmosavi et al 2013]. Wurzinger et al (2009) discute o uso de serviços web para o desenvolvimento de Ecossistemas de E-learning mais flexíveis construídos a partir de composição de recursos educacionais, chamados de serviços, utilizando conceitos de Arquitetura Orientada a Serviços (SOA). Karavirta et al (2013) utiliza uma abordagem de SOA para melhorar a interoperabilidade de sistemas de e-learning, através de serviços web REST, que permite a integração de ferramentas externas para realização de avaliações e exercícios.

Uma alternativa para interoperar dados de diferentes origens dentro do ecossistema é por meio do uso de repositórios de informações de experiências de aprendizagem, incluindo jogos, é uma característica do xAPI (antes TinCan API) [ADL 2013], proposto como a próxima geração do SCORM [ADL 2015] pela ADL. Essas experiências seguem um formato baseado na ação (ler, completar, iniciar) de atores (estudantes, grupos) sobre objetos (lições, conteúdos, mídias) e são armazenadas em Repositórios de Registros de Aprendizagem (Learning Record Store- LRS) por serviços web REST.

Embora existam trabalhos incorporando gamificação à AVAs [Del Blanco et al 2011][Kiryakovam et al. 2014], que podem ser plataformas a partir da qual Ecossistemas de E-learning se estabelecem, não se identificou na literatura, até o momento, o estudo de gamificação em e-learning sob a perspectiva de Ecossistemas de E-learning. LUDOS não propõe um novo Ecossistema de E-learning, mas uma infraestrutura para integrar conteúdo educacional, serviços e aplicações com gamificação a ecossistemas existentes através de padrões de distribuição de conteúdo educacional. A proposta prioriza a criação de serviços que permitam a criação e adoção de novas ferramentas, o estabelecimento de novos padrões, fornecedores $\mathrm{e}$ possibilidades de interação entre as comunidades existentes, com o objetivo de diminuir 
as barreiras para a adoção de gamificação nesses ecossistemas. A seção 4 detalha a proposta da LUDOS, ressaltando estas características.

\section{Infraestrutura LUDOS}

A infraestrutura LUDOS, do latim ludos, que significa jogos e diversão, foi criada com o objetivo de facilitar o uso de gamificação em Ecossistemas de E-learning. Para atender a esse objetivo, foi definida uma arquitetura baseada em serviços web para armazenamento e consulta de dados relacionados a jogos e elementos de jogos.

Os dados armazenados estão relacionados a mecanismos de jogos presentes nos OAs para recompensar e dar visibilidade de progresso, como pontos obtidos ao concluir um teste, uma medalha ao concluir um desafio ou um título por completar atividades opcionais. Vamos nos referir a essas recompensas obtidas durante a interação com OAs sempre como conquista. A partir desses dados é possível a criação de serviços e ferramentas no ecossistema que favoreçam o engajamento e a motivação dos estudantes, como páginas de perfis sociais com histórico de conquistas, quadros de líderes, acompanhamento de metas individuais e coletivas.

Com a infraestrutura LUDOS, as características originais dos ecossistemas são mantidas, e suas fronteiras são ampliadas para incluir novos componentes, tanto bióticos (provedores de conteúdo com características de jogos, desenvolvedores e comunidades de código livre para compartilhamento de aplicações e serviços educacionais baseados em jogos) quanto abióticos (ferramentas de autoria, OAs com características de jogos, repositórios de conquistas, novas aplicações e serviços baseados em jogos) (Figura-1).

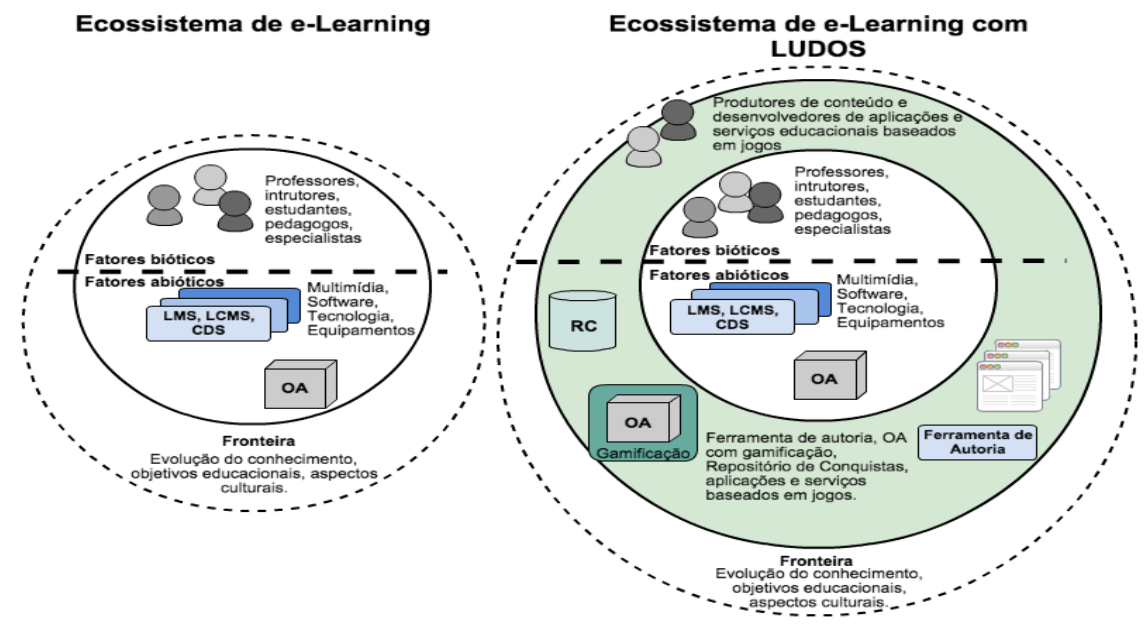

Figura 1. Fatores de um Ecossistema de E-learning antes e após a LUDOS.

\subsection{Arquitetura}

A arquitetura da LUDOS é baseada em serviços web REST, com dois objetivos principais: i) permitir a coleta de dados gerados durante a interação dos estudantes com os OAs e; ii) sua consulta por aplicações e serviços dentro do Ecossistema de Elearning, levando em conta a segurança, privacidade e diferentes níveis de acesso.

Para facilitar a construção de OAs com as características necessárias para o envio de informações de conquistas, a LUDOS define uma Ferramenta de Autoria que, embora não seja um componente obrigatório, permite a criação de OA com características de jogos sem a necessidade de conhecimento técnico, visto que esses recursos frequentemente são criados por professores sem esse conhecimento [Silveira e Carneiro 2012]. A Ferramenta de Autoria também permite que um mesmo OA seja 
gerado em diferentes padrões, permitindo que um fornecedor de conteúdo disponibilize o mesmo conteúdo educacional em diferentes Ecossistemas de E-learning. $\mathrm{Na}$ Ferramenta de Autoria, os elementos de jogos podem ser associados a interações com o estudante como resolução de atividades, testes e progresso no conteúdo. É importante ressaltar que metadados, conteúdo multimídia e outros tipos de conteúdo educacional tradicional também são suportados, e é a partir deles que estão definidos os elementos de gamificação.

As informações de conquistas obtidas pelos estudantes são enviadas para um repositório de dados de gamificação chamado Repositório de Conquistas (RC), com uma arquitetura em camadas (Figura-2a), onde os dados são armazenados e disponibilizados através de uma API via serviços web REST (Figura-2b). O RC, embora tenha um pequeno conjunto de informações obrigatórias para identificar estudantes, organizações e OA, permite a persistência e recuperação de informações adicionais relacionadas às conquistas, dando flexibilidade para que necessidades específicas de um Ecossistema de E-learning ou aplicação sejam atendidas.

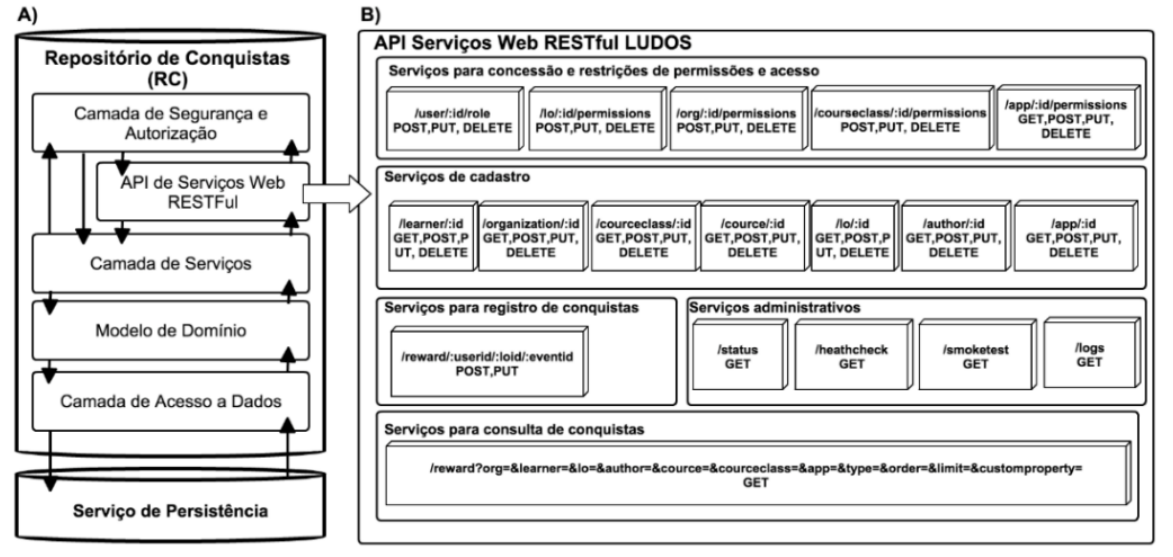

Figura 2. A) Camadas do Repositório de Conquistas (RC). B) Recursos e verbos disponíveis na API.

A LUDOS abrange as etapas desde a criação do OA, com as extensões necessárias, o armazenamento dessas informações no RC, e sua disponibilização para aplicações e serviços educacionais baseados em gamificação, de modo que: i) o professor crie o OA (Figura-3A) e o disponibilize no ecossistema (Figura-3B); ii) O estudante interaja com o OA (Figura-3C), enviando dados tradicionais para o LMS e os dados de gamificação para o RC (Figura-3D); iii) estes dados podem ser consultados pela plataforma educacional (Figura-3E) ou iv) por outras aplicações e serviços presentes no ecossistema (Figura-3F).

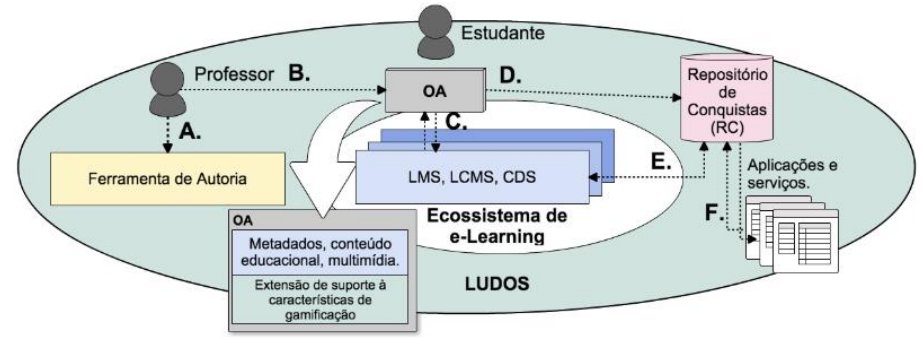

Figura 3. Componentes da infraestrutura LUDOS.

\subsection{Integração com Ecossistemas de E-learning}

A LUDOS não define como a gamificação é utilizada no ecossistema, mas define uma infraestrutura que pode ser facilmente incorporada, e uma API de serviços web que 
permite que ecossistemas diferentes compartilhem aplicações, serviços e recursos construídos com base nesses serviços. Mesmo em um cenário em que o esforço para adoção da LUDOS seja comparável à criação de uma solução própria para a adição de gamificação ao ecossistema, a LUDOS oferece uma vantagem significativa: a possibilidade de interoperabilidade de dados, serviços e aplicações educacionais com características de jogos construídos a partir de sua API.

\section{Metodologia de Desenvolvimento}

Com o objetivo de servir como uma Prova de Conceitos (POC), uma primeira versão da infraestrutura LUDOS foi implementada e utilizada para disponibilização de OAs com suporte a gamificação em um AVA. Assim, essa abordagem de uso da LUDOS têm como objetivo permitir sua avaliação e para isso, a seguinte metodologia de desenvolvimento foi adotada: 1) escolha de um padrão de OA sem suporte nativo para envio de dados para serviços web, e extensão do mesmo para o envio de dados de conquistas; 2) desenvolvimento da Ferramenta de Autoria para facilitar a criação de OA com a extensão necessária; 3) desenvolvimento do RC para armazenar os dados obtidos a partir dos OAs estendidos; 4) criação de uma aplicação independente alimentada a partir dos dados armazenados no RC; e 5) reprodução do OA gerado em um AVA sem necessidade de alterações no mesmo.

\subsection{Escolha de um padrão de OA}

A escolha do padrão de OA utilizado para a avaliação foi feita com base nos seguintes critérios: ser um padrão adotado pela indústria, amplamente documentado e que possa ser utilizado, testado e validado de forma gratuita. Foi selecionado o padrão SCORM[ADL 2015], um modelo de referência para empacotamento de OA baseado em recomendações e especificações. No contexto deste trabalho foi desenvolvida uma extensão para ser incorporada aos OA em formato SCORM para criação de uma camada para captura de eventos de progresso do estudante durante sua interação com o mesmo. Com base em um mapeamento em um arquivo XML, cada um desses avanços (conteúdo visualizado, lição respondida, desafio completado) é associado a uma conquista (pontos, medalhas, títulos), e enviada para um ou vários serviços web externos.

\subsection{Ferramenta de Autoria}

A Ferramenta de Autoria é uma aplicação web que permite a criação de OAs incluindo conteúdo educacional, metadados, mídias e, além disso, a extensão para suporte à gamificação, desenvolvida na etapa anterior. A criação dos OA através da Ferramenta de Autoria é feita por meio de seleção de características com base em um modelo de modelo de features (Figura-4A), que é uma forma de descrever características obrigatórias, opcionais e variantes de uma família de aplicações relacionadas a um dado domínio, neste caso recursos educacionais na forma de OA. Está fora do escopo deste trabalho o detalhamento do modelo de features (Figura-4B), que faz parte de uma abordagem de Linha de Produto de Software Educacional, desenvolvida no contexto do projeto BROAD [Campos et al, 2012]. A cada característica selecionada novas opções complementares tornam-se disponíveis. Cada opção de preenchimento obrigatório ainda não realizada é exibida como um alerta, assim como configurações inconsistentes ou conflitantes. O OA é gerado quando todas as características obrigatórias forem selecionadas. Entre as características disponíveis estão aquelas associadas a jogos, como pontos e medalhas, que podem ser associadas ao progresso do estudante no conteúdo disponível no OA. 


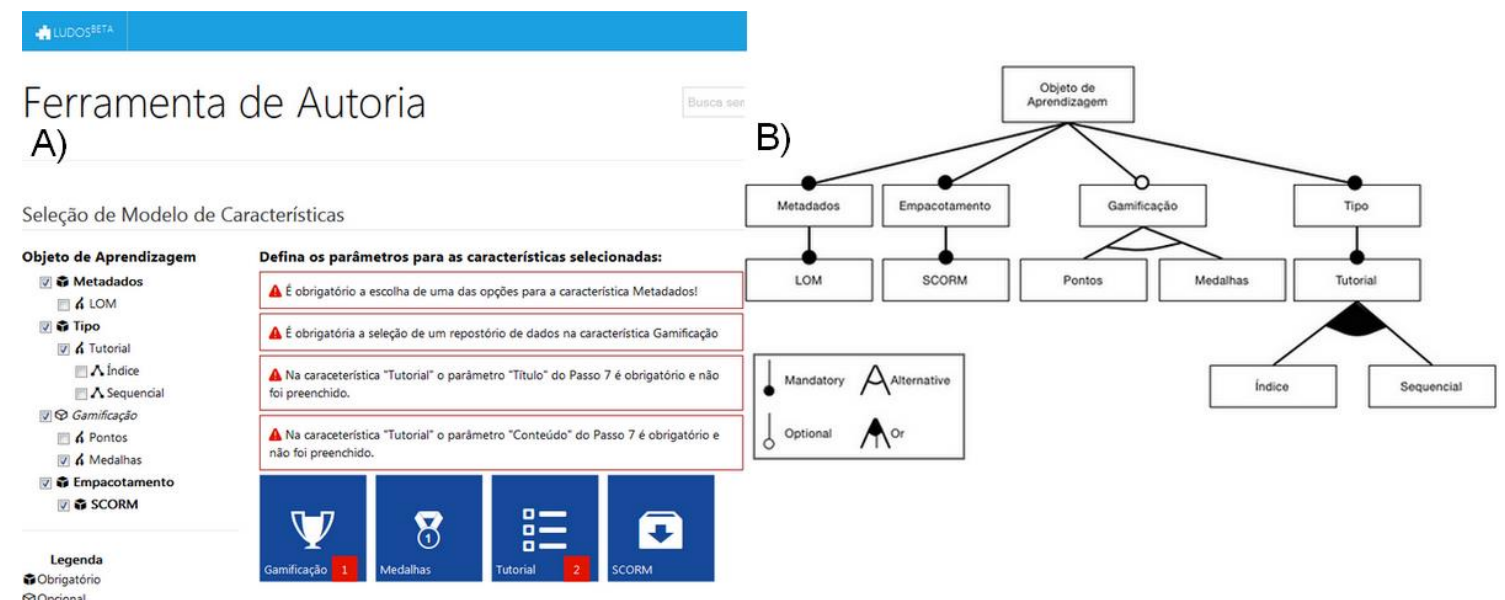

Figura4. A) Interface para criação de OAs (B) Modelo de Features.

\subsection{Repositório de Conquistas}

$\mathrm{O} \mathrm{RC}$ foi desenvolvido na linguagem Java, como um serviço à parte, utilizando o banco de dados não relacional, orientado a documentos, MongoDB ${ }^{1}$, para persistência. A versão atual possui todas as camadas definidas na arquitetura. No entanto, foram desenvolvidos para a Prova de Conceitos, apenas os serviços necessários para a avaliação funcional da infraestrutura, ou seja, os serviços para registro e consulta de conquistas e um subconjunto dos serviços de cadastro.

\subsection{Rankr}

O objetivo do desenvolvimento do Rankr é demonstrar como os dados gerados durante a interação dos estudantes com os $\mathrm{OA}$, podem ser consolidados para a criação de aplicações independentes, baseadas em elementos de jogos. O Rankr tem como objetivo adicionar ao ecossistema, regras, visibilidade, dinâmica ou recursos de jogos.

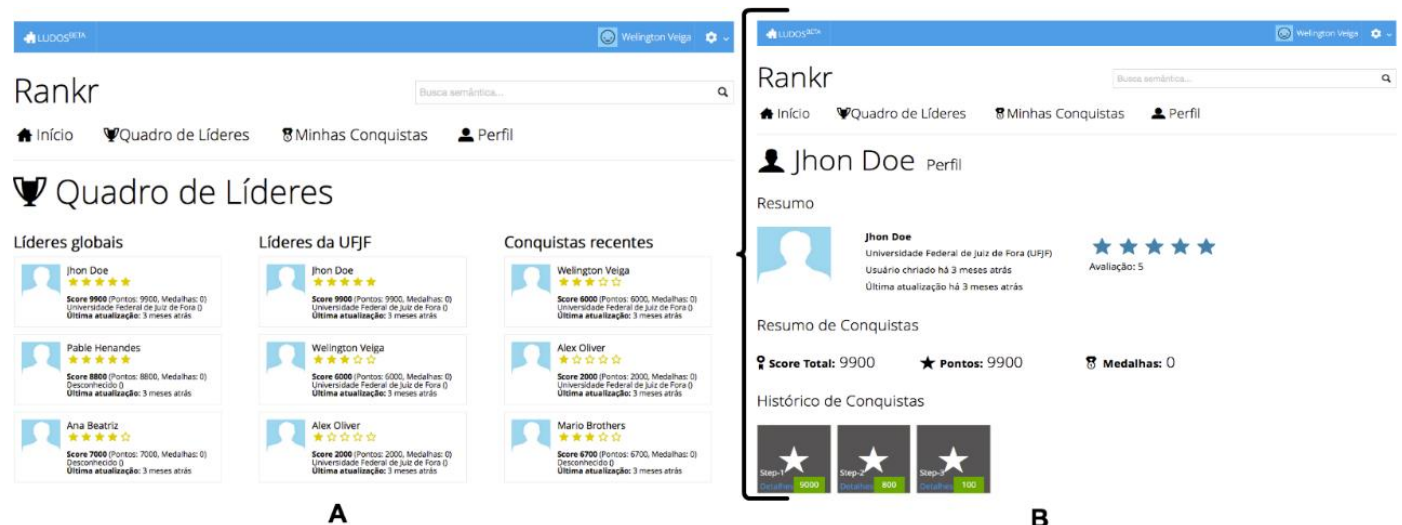

Figura7. Rankr, aplicação para exibição de Quadro de Líderes (A), conquistas do próprio estudante e perfis com detalhes do histórico de pontuação (B).

Desta forma, o Rankr é uma aplicação para exibição de perfis sociais de estudantes em um Quadro de Líderes, dividido em três partes: os estudantes com mais pontos, os estudantes com mais pontos pertencentes à mesma organização que o estudante autenticado e as últimas conquistas obtidas (Figura-7A). Também é possível visualizar em quais cursos/OAs ele obteve suas conquistas (Figura-7B). Está também presente o conceito de recompensa com base em estrelas, onde os estudantes possuem um número proporcional de estrelas de acordo com seu desempenho.

${ }^{1}$ https://www.mongodb.org/ 


\subsection{Avaliação da proposta e análise dos resultados}

Para avaliar os requisitos funcionais do LUDOS foi então definido um cenário de uso (Wohlin et al., 2012), com o objetivo de avaliar a infraestrutura LUDOS, considerando a integração a um AVA, como plataforma de um Ecossistema de E-learning. O cenário de uso foi assim definido: 1) Utilização de uma plataforma de código livre; 2) A plataforma, os componentes da infraestrutura LUDOS e demais serviços utilizados estariam disponíveis em nuvem; 3) Seriam identificados atores pertencentes ao ecossistema selecionado; 4) Um roteiro seria definido, evidenciando os fatores bióticos e abióticos envolvidos em cada etapa; 5) As etapas do roteiro seriam implementadas e as observações do ponto de vista dos atores registrados.

A plataforma escolhida para o cenário de uso foi o Moodle, um LMS de código aberto, utilizado em dezenas de milhares de ambientes de e-learning em todo mundo [Moodle 2015], e configurado em uma infraestrutura na nuvem para disponibilizar separadamente o Moodle, a Ferramenta de Autoria, o RC e o Rankr. Os atores, o roteiro e a avaliação realizada estão detalhados na Tabela-1.

\begin{tabular}{|c|c|c|c|}
\hline \multicolumn{3}{|c|}{ Roteiro de avaliação } & Resultados \\
\hline Etapa & Descrição & Atores & Observações \\
\hline $\begin{array}{l}\text { 1. Criação do } \\
\text { OA na } \\
\text { Ferramenta de } \\
\text { Autoria }\end{array}$ & $\begin{array}{l}\text { Produção de um conteúdo } \\
\text { educacional do tipo tutorial em } \\
\text { formato SCORM, sobre Malária, } \\
\text { utilizando recursos } \\
\text { desenvolvidos em Campos et al } \\
\text { (2012): }\end{array}$ & Professor/Conteudista & $\begin{array}{l}\text { A Ferramenta de Autoria facilitou a criação do } \\
\text { OA, o único detalhe técnico necessário foi o } \\
\text { endereço a partir do qual o RC estaria } \\
\text { disponível. } \\
\text { Em algumas etapas do tutorial foram associadas } \\
\text { medalhas e pontos conquistados pelo estudante } \\
\text { que as concluir. }\end{array}$ \\
\hline $\begin{array}{l}\text { 2. Criação do } \\
\text { curso no Moodle }\end{array}$ & $\begin{array}{l}\text { Criação do curso "Malária " na } \\
\text { plataforma Moodle, onde foi } \\
\text { disponibilizado o OA em } \\
\text { formato SCORM gerado pela } \\
\text { ferramenta de autoria. }\end{array}$ & $\begin{array}{l}\text { Administrador da } \\
\text { Plataforma }\end{array}$ & $\begin{array}{l}\text { Nenhuma mudança em relação à distribuição de } \\
\text { conteúdo sem gamificação. }\end{array}$ \\
\hline $\begin{array}{l}\text { 3atrícula de } \\
\text { um estudante no } \\
\text { curso. }\end{array}$ & $\begin{array}{l}\text { Matrícula de um estudante na } \\
\text { plataforma Moodle e no curso } \\
\text { criado. }\end{array}$ & $\begin{array}{l}\text { Administrador da } \\
\text { Plataforma }\end{array}$ & $\begin{array}{l}\text { Nenhuma mudança em relação à matrícula para } \\
\text { um curso sem gamificação. }\end{array}$ \\
\hline 4. Acesso ao OA & $\begin{array}{l}\text { Registro da interação do } \\
\text { estudante com o OA } \\
\text { disponibilizado no curso. }\end{array}$ & Estudante & $\begin{array}{l}\text { O acesso ao curso e ao OA mantem-se o } \\
\text { mesmo. } \\
\text { À medida que as etapas com conquistas } \\
\text { associadas foram cumpridas, mensagens de } \\
\text { sucesso com a medalha obtida foram exibidas. }\end{array}$ \\
\hline $\begin{array}{l}\text { 5. Consulta aos } \\
\text { dados no Rankr }\end{array}$ & $\begin{array}{l}\text { Verificação dos resultados } \\
\text { obtidos pelo estudante no Rankr. }\end{array}$ & Estudante/Professor & $\begin{array}{l}\text { O progresso dos estudantes é exibido no Rankr, } \\
\text { onde o estudante pode visualizar seu progresso, } \\
\text { compará-lo com os dos colegas e os melhores } \\
\text { de todo ecossistema, bem como acompanhar as } \\
\text { conquistas recentes. } \\
\text { O professor pode acompanhar o progresso dos } \\
\text { estudantes. }\end{array}$ \\
\hline
\end{tabular}

Tabela 1. Roteiro para avaliar a LUDOS e a integração com o LMS Moodle.

Embora o desenvolvimento da infraestrutura ainda esteja em andamento, a versão atual permitiu a especificação do cenário de uso e a análise de viabilidade de, com a infraestrutura LUDOS, adicionar gamificação em Ecossistemas de E-learning com alterações mínimas. Vimos também a viabilidade de se criar e adicionar aplicações voltadas a gamificação com o Rankr.

É importante destacar, portanto, que a LUDOS atende às características básicas necessárias para a adoção de gamificação em Ecossistemas de E-learning do ponto de vista técnico, oferecendo a infraestrutura necessária. Para o sucesso da adoção de gamificação em um ecossistema, a construção de um conteúdo coerente com os objetivos educacionais e ao mesmo tempo rico do ponto de vista de gamificação é essencial e, dessa forma, os resultados aqui apresentados precisam de ser posteriormente validados a partir de um modelo de experimentação formal e mais abrangente. No 
entanto, estes resultados mostram indícios da adequabilidade da abordagem e fornecem subsídios para novas avaliações.

\section{Considerações finais e trabalhos futuros}

Este trabalho apresentou uma abordagem para viabilizar o uso de gamificação em Ecossistemas de E-learning, sem necessidade de alterações profundas na plataforma e enriquecendo o ecossistema com a incorporação de novos atores, aplicações e interações. O modelo de desenvolvimento adotado, os testes realizados e o cenário de uso permitiram que obtivéssemos indícios da aplicabilidade da infraestrutura e a possibilidade de incorporar novas aplicações voltadas à gamificação a ecossistemas.

Como trabalhos futuros, estão a continuidade do desenvolvimento da infraestrutura LUDOS, incluindo a evolução em relação a algumas limitações da implementação utilizada no escopo deste trabalho, e a ampliação do instrumento de avaliação da infraestrutura, através de experimentos mais formais como estudos de casos em ambientes de e-learning na academia e na indústria sob a perspectiva de Ecossistemas de E-learning, entrevistando os diferentes atores nesses cenários para capturar sua percepção, validando a proposta em contextos mais abrangentes.

\section{Referências}

ADL (2015) "SCORM: CAPABILITY INFORMATION" disponível em http://www.adlnet.gov/scorm./, acessado em 25 de Fevereiro de 2015.

ADL (2013) "Experience API". Disponível em: http://tincanapi.com/. Acessado em 15 de Março de 2015.

Alario-Hoyos, C.; Bote-Lorenzo, M.; Gómez-Sánchez, E.; Asensio-Pérez, J.; VegaGorgojo, G. e Ruiz-Calleja, A. (2013). "GLUE!: An architecture for the integration of external tools in Virtual Learning Environments". Comp. and Education, 60(1).

Amriani, A.; Aji, A. ; Utomo, A. e Junus, K. (2013) "An empirical study of gamification impact on e-Learning environment." Computer Science and Network Technology (ICCSNT), 2013 3rd International Conference on. IEEE, 2013.

Borges, S. S., Reis, H. M., Durelli, V. H. S., Bittencourt, I. I., Jaques, P. \& Isotani, S. (2014). "A systematic mapping on gamification applied to education" in Proceedings of the 29th Annual ACM Symposium on Applied Computing. p. 216-222.

Campos, F.; Braga, R.; Nery, T. e Santos, N. (2012) "Rede de Ontologias: apoio semântico a linha de produtos de objetos de aprendizagem" An. do Simpósio Bras. Informática na Educ., vol. 23, no. SBIE, pp. 26-30.

Del Blanco, A.; Torrente, J.; Moreno-Ger, P. e Fernández-Manjón, B. (2011). "Enhancing adaptive learning and assessment in virtual learning environments with educational games". IJDET: Emerging Studies, 144-163.

Dicheva, D.; Dichev, C.; Agre, G.; e Angelova, G. (2015). "Gamification in education: A systematic mapping study". Educational Technology and Society, p. 18(3).

Docebo, 2014. "E-Learning Market Trends \& Forecast 2014-2016", Docebo Report. Disponível em http://tinyurl.com/docebo-report. Acesado em 15de março de 2015.

Doelitzscher, F. Sulistio, A.; Reich, C.; Kuijs, H. e Wolf, D. (2011) "Private cloud for collaboration and e-Learning services: from IaaS to SaaS". Computing 91, p. 23-42. 
Domínguez, A., Saenz-de-Navarrete, J., de-Marcos, L., Fernández-Sanz, L., Pagés C., and Martínez-Herráiz, J. (2013). "Gamifying learning experiences: Practical implications and outcomes". Computers and Education. 63, 380-392.

Glover, I. (2013) "Play as you learn: gamification as a technique for motivating learners". In: Proceedings of World Conference on Educational Multimedia, Hypermedia and Telecommunications 2013.

Guetl C. and Chang, V. (2008) "The use of Web 2.0 Technologies and Services to support E-Learning Ecosystem to develop more effective Learning Environments", the Ninth IEEE International Conference on Advanced Learning Technologies.

IMS GLC (2006) "IMS Tool Interoperability Guidelines". Version 1.0. Disponível em: http://www.imsglobal.org/ti/. Acessado em 15 de Março de 2015

Kapp, K. (2012). "The gamification of learning and instruction: Game-based methods and strategies for training and education". San Francisco, CA: Pfeiffer.

Kiryakovam G.; Angelova, G. e Yordanov, L. (2014) "Gamification in education". Proceedings of 9th International Balkan Education and Science Conference.

Lohmosavi, V.; Nejad, A. e Hosseini, E., (2013) "E-learning ecosystem based on service-oriented cloud computing architecture," Information and Knowledge Technology (IKT), 2013 5th Conference on, vol., no., pp.24,29, 28-30 May 2013

Medeiros, C; Fernandes, A.; Damasceno, F. (2014) "Uma Abordagem Gamificada para Prevenção do uso de Drogas com Adolescentes". In Anais do Simpósio Brasileiro de Informática na Educação (SBIE 2014)

Moodle (2015) "Moodle Statistics". Disponível em https://moodle.net/stats/. Acessado em 10 de Maio de 2015.

Muntean, C. I. (2011). "Raising engagement in e-learning through gamification". In Proc. 6th International Conference on Virtual Learning ICVL (pp. 323-329).

Nery, T.; Campos, F.; Braga, R.; Santos, N. e Mattos, K. (2012) "BROAD Project: Semantic Search and Application of Learning Objects," IEEE Technol. Eng. Educ.

Pereira. C.; Campos, F.; Ströele, V.; David, M. N. e Braga, R. "Extração de Características de Perfil e de Contexto em Redes Sociais para Recomendação de Recursos Educacionais,” XXV Simpósio Bras. Informática na Educ. (SBIE 2014).

Sridharan, B., Deng, H., Corbitt, B. (2010),"Critical success factors in e\#learning ecosystems: a qualitative study", Journal of Systems \& Inf. Technology, p 263 - 288.

Silveira, M. S. and Carneiro, M. L. F. (2012). "Desconstruindo Objetos de Aprendizagem: reflexões sobre sua qualidade de uso". In Anais do XXIII Simpósio Brasileiro de Informática na Educação (SBIE 2012).

Wohlin, C.; Runeson, P.; Höst, M.; Ohlsson, M.; Regnell, B. e Wesslén A. (2012) "Experimentation in Software Engineering". Springer Berlin Heidelberg, 2012.

Wurzinger, G., Chang, V.r Guetl, C. (2009). "Towards greater flexibility in the learning ecosystem - Promises and obstacles of service composition for learning environments". IEEE Int. Conf. on Digital Ecosystems \& Technologies, DEST '09. 